\title{
Profiling analysis of circulating microRNA expression in cervical cancer
}

\author{
YUZO NAGAMITSU, HIROTAKA NISHI, TORU SASAKI, \\ YOTARO TAKAESU, FUMITOSHI TERAUCHI and KEIICHI ISAKA
}

Department of Obstetrics and Gynecology, Tokyo Medical University, Tokyo 160-0023, Japan

Received December 18, 2015; Accepted April 18, 2016

DOI: $10.3892 / \mathrm{mco} .2016 .875$

\begin{abstract}
MicroRNA (miRNA) expression is altered in cancer cells and is associated with the development and progression of various types of cancer. Accordingly, miRNAs may serve as diagnostic or prognostic biomarkers in cancer patients. In this study, we attempted to analyze circulating exosomal miRNA in patients with cervical cancer. Total RNA was extracted from the serum of healthy subjects, subjects with cervical intraepithelial neoplasia (CIN) and patients with cervical cancer. We first investigated miRNA expression profiles in 6 serum samples from healthy subjects and patients with cervical cancer using the miRCURY LNA microRNA array. miRNAs with significant differences in expression were validated in a larger sample set by quantitative reverse transcription-polymerase chain reaction, using TaqMan gene expression assays. The results of the miRCURY LNA microRNA array indicated that 6 of 1,223 miRNAs found in serum samples from cervical cancer patients and normal controls exhibited a $>3.0$-fold change in expression level in subjects with cervical cancer, with a P-value of $<0.01$. In a validation set $(n=131)$ that investigated the expression of 4 of the 6 miRNAs (miR-483-5p, miR-1246, miR-1275 and miR-1290), miR-1290 was found to have significantly higher expression levels in cervical cancer samples $(n=45)$ compared with control samples $(n=31)$. We also found that the median levels of these miRNAs were significantly higher in subjects with cervical cancer $(n=45)$ compared with those in subjects with CIN $(n=55)$. Circulating miRNAs were not correlated with clinicopathological parameters. However, receiver operating characteristic curve analysis suggested that these serum miRNAs may be useful diagnostic markers in cervical cancer. The expression of circulating miR-1290 was significantly higher in the blood of cervical cancer patients compared with that in controls and may thus serve as a useful biomarker in cervical cancer diagnosis. However, larger studies are required
\end{abstract}

Correspondence to: Dr Hirotaka Nishi, Department of Obstetrics and Gynecology, Tokyo Medical University, 6-7-1 Nishishinjuku, Shinjuku-ku, Tokyo 160-0023, Japan

E-mail: nishih@tokyo-med.ac.jp

Key words: biomarker, microRNA, cervical cancer, miR-1290 to fully elucidate the role of circulating exosomal miRNAs in cervical cancer.

\section{Introduction}

Cervical cancer is the second most common cancer in women worldwide after breast cancer, and it is particularly prevalent in developing countries (1), accounting for $\sim 275,100$ deaths annually worldwide (2). Cervical cancer is caused by persistent infection by high-risk types of the human papillomavirus (HPV) (3). Histologically, cervical cancer is almost entirely classified into two types: Squamous cell carcinoma (SCC; 80\%) and adenocarcinoma ( 5-20\%) (4). SCC develops from cervical intraepithelial neoplasia (CIN), whereas adenocarcinoma develops from intraepithelial adenocarcinoma and glandular dysplasia. To reflect their relative risk of progression to cervical cancer, CIN1 is considered low-grade CIN, whereas CIN2-3 is considered high-grade CIN. High-grade CIN may arise 2-3 years after high-risk HPV infection, subsequently causing cervical cancer after $\geq 10$ years (5).

The most widely known tumor marker for cervical cancer is SCC antigen, which is a tumor-associated antigen identified by Kato et al in 1977 (6). The positive detection rate of SCC antigen in each clinical stage of cervical cancer is $2.4 \%$ (stage 0), 22.2\% (stage I), 56.7\% (stage II), 76.4\% (stage III), $76.9 \%$ (stage IV) and $87 \%$ in recurrent cancer (7). However, the positive detection rate is low in early stages.

MicroRNAs (miRNAs) are small (typically 19-25 nucleotides), non-coding, endogenous, single-stranded RNAs. miRNAs were first described in 1993 by Lee et al in Caenorhabditis elegans (8). The majority of miRNAs negatively regulate target mRNAs in diverse biological processes, including cell proliferation, differentiation, development, metabolism and death. miRNAs are often aberrantly expressed in a number of human malignancies, and play key roles in tumor initiation and development. miRNAs suppress the translation of target mRNAs, mainly by binding to their 3'-untranslated region (9). Moreover, miRNAs significantly affect the expression of tumor oncogenes and suppressor genes, being involved in both tumor promotion and tumor suppression. Furthermore, epigenetic changes, such as DNA methylation, are involved in the abnormal expression of certain miRNAs $(2,10)$. A single miRNA may have thousands of targets and approximately one-third of human genes may be controlled by miRNAs (11). 
The mechanism of miRNA expression in cancer cells has not been fully elucidated. However, altered expression of miRNAs has been detected in various types of cancer and appears to play a major role in the onset and progression of cancer.

It was recently discovered that miRNAs embedded in the small granules of exosomes are secreted into extracellular regions, including serum, urine and saliva (12). Serum miRNA detection may serve as a diagnostic or prognostic biomarker in cancer patients. Accordingly, our study was designed to analyze circulating miRNA levels in subjects with cervical cancer, in order to assess the potential value of miRNAs as diagnostic biomarkers.

\section{Materials and methods}

Study design and study population. A total of 131 subjects participated in the study, which was conducted at the Tokyo Medical University Hospital from April, 2010 to March, 2012. Of these subjects, 45 had cervical cancer, 55 had CIN and 31 were healthy. The research protocol was approved by our Institutional Review Board (approval no. 3268) and informed consent was obtained from all the subjects. Of the 45 patients with cervical cancer, 7 were stage Ia, 16 were stage Ib, 10 were stage IIb, 3 were stage III and 2 were stage IV. Of the 55 subjects with CIN, 15 were CIN1, 16 were CIN2 and 24 were CIN3. The patient backgrounds were obtained through interviews. Blood samples were collected prior to chemotherapy and radiation therapy. Cervical cancer and CIN were diagnosed on the basis of histological examinations. The characteristics of the subjects are summarized in Table I.

The present study was conducted in three phases (Fig. 1). In the first phase (screening phase), we performed comprehensive analysis of the serum miRNA of the cervical cancer subjects using a microarray, and selected 6 candidate miRNAs. We used 10 samples in total, 5 from cervical cancer subjects and 5 from healthy subjects. In the second phase (selection phase), the 6 candidate miRNAs were narrowed down to 4 using reverse transcription-quantitative polymerase chain reaction (RT-qPCR). For the RT-qPCR, we used serum miRNA from 20 subjects with cervical cancer and 10 healthy subjects. In the third phase (validation phase), we performed RT-qPCR on the 4 miRNAs identified during the selection phase. In order to validate these 4 miRNAs, we examined the serum miRNA of 45 subjects with cervical cancer, 55 subjects with CIN and 31 healthy subjects, performed statistical processing and investigated the potential of each miRNA as a tumor marker.

Comprehensive analysis of serum miRNAs by microarray. In the screening phase, total RNA was extracted using ISOGEN-LS (Nippon Gene, Tokyo, Japan) and the serum of 5 subjects with cervical cancer and that of 5 healthy subjects. Total RNAs were purified using a column. Each total RNA was labeled with Hy3 or Hy5 using high-affinity locked nucleic acid (LNA) probes, hybridized overnight and scanned to be digitized. We selected the miRNAs exhibiting an expression level in the cervical cancer subjects that was more than three times higher compared with that in healthy subjects, as indicated by miRNA profiling, which left us with 6 miRNA species out of the original 1,223.
Identification of 4 miRNA species by qPCR. In the selection phase, total RNA was extracted from the serum of 20 subjects with cervical cancer and 10 healthy subjects. RT-qPCR was performed on the total RNA, and 4 miRNA species were selected (miR-483-5p, miR-1246, miR-1275 and miR-1290) from the 6 miRNA species. In the validation phase, multiple samples were used to validate the miRNAs. Total RNA was extracted from the serum of 45 subjects with cervical cancer, 55 subjects with CIN and 31 healthy subjects, and subjected to RT-qPCR.

Serum preparation and total RNA extraction. Venous blood samples were collected from cervical cancer patients and healthy controls. The samples were separated into blood cells and serum by centrifugation, and stored at $-5^{\circ} \mathrm{C}$. Total RNA in the serum was isolated using ISOGEN-LS, according to the manufacturer's instructions. Briefly, $250 \mu \mathrm{l}$ of serum was homogenized in $750 \mu \mathrm{l}$ of ISOGEN-LS and $200 \mu \mathrm{l}$ of chloroform was added to the sample, which was centrifuged. Following additional chloroform extraction and precipitation with isopropanol, the RNA sample was suspended in $20 \mu \mathrm{l}$ of nuclease-free water (13).

Validation of miRNA expression by qPCR. We quantified miRNAs using TaqMan MicroRNA assays (Applied Biosystems, Foster City, CA, USA) with modifications and miRNA-specific stem-loop primers (has-miR-1290; Applied Biosystems). First, each miRNA was specifically reverse-transcribed to cDNA according to the manufacturer's protocol, using TaqMan miRNA RT-Kit with stem-loop RT-primer and the Applied Biosystems 9800 Fast Thermal Cycler. Second, qPCR was performed for each specific miRNA using an RT-primer with Universal Master Mix on the Applied Biosystems StepOnePlus ${ }^{\mathrm{TM}}$ Real-Time PCR system (Life Technologies Corporation, Carlsbad, CA, USA). Sequence detection was performed according to the manufacturer's protocol. The reaction mixtures were incubated at $95^{\circ} \mathrm{C}$ for $2 \mathrm{~min}$, followed by 50 cycles at $95^{\circ} \mathrm{C}$ for $15 \mathrm{sec}$ and $60^{\circ} \mathrm{C}$ for $1 \mathrm{~min}$. Each sample was analyzed in duplicate. The cycle thresholds $(\mathrm{Ct})$ for subjects with cervical cancer, subjects with CIN and healthy subjects were calculated and normalized to miR-16, which was found in the literature to be the most widely-used endogenous control miRNA for RT-qPCR (14). The expression levels of miRNAs in subjects with cervical cancer and those with CIN relative to healthy controls were calculated using the comparative $\mathrm{Ct}$ method. The average $\mathrm{Ct}$ value of the control miR-16 for every sample was subtracted from the $\mathrm{Ct}$ value for each respective mature miRNA reaction, resulting in the $\Delta \mathrm{Ct}$ value. The $-\Delta \Delta \mathrm{Ct}$ value was calculated by subtracting the $-\Delta \mathrm{Ct}$ value of a normal sample from the respective $-\Delta \mathrm{Ct}$ values of the patient samples. Expression of miR-1290 was normalized using the $2^{-\Delta \Delta \mathrm{Ct}}$ method $(13,14)$. The miR-1290 expression profile was used to create a receiver operating characteristic (ROC) curve, which is a graphical plot of the true-positive vs. the false-positive rate. The area under the ROC curve represents the discrimination accuracy.

Statistical analysis. Statistical analysis of the causal association between the clinical background and expression level of the miRNAs was performed using EZR software (15). A 
Table I. Characteristics of the subjects participating in the study.

\begin{tabular}{|c|c|c|c|c|c|}
\hline Characteristics & $\begin{array}{l}\text { Cervical cancer } \\
\qquad(n=45)\end{array}$ & $\begin{array}{l}\text { CIN1 } \\
(n=15)\end{array}$ & $\begin{array}{l}\text { CIN2 } \\
(n=16)\end{array}$ & $\begin{array}{l}\text { CIN3 } \\
(n=24)\end{array}$ & $\begin{array}{l}\text { Normal } \\
(n=31)\end{array}$ \\
\hline \multicolumn{6}{|l|}{ Age, years } \\
\hline Median \pm SD & $49.0 \pm 14.1$ & $34.0 \pm 6.7$ & $35.0 \pm 9.2$ & $34.0 \pm 4.9$ & $39.0 \pm 11.2$ \\
\hline Range & 28-88 & $27-50$ & $19-55$ & $26-43$ & 24-69 \\
\hline Smoking & 9 & 3 & 5 & 3 & 3 \\
\hline \multicolumn{6}{|l|}{ Histological type } \\
\hline $\mathrm{SCC}$ & 38 & & & & \\
\hline Adenocarcinoma & 9 & & & & \\
\hline \multicolumn{6}{|l|}{ Clinical stage } \\
\hline Ia & 7 & & & & \\
\hline $\mathrm{Ib}$ & 16 & & & & \\
\hline IIa & 7 & & & & \\
\hline $\mathrm{IIb}$ & 10 & & & & \\
\hline IIIIV & 3 & & & & \\
\hline IV & 2 & & & & \\
\hline \multicolumn{6}{|c|}{ Serum $\mathrm{SCC}$ antigen, $\mathrm{ng} / \mathrm{ml}$} \\
\hline Median \pm SD & $2.65 \pm 6.61$ & & & & \\
\hline
\end{tabular}

CIN, cervical intraepithelial neoplasia; SD, standard deviation; SCC, squamous cell carcinoma.

Screening phase

\begin{tabular}{l}
$\begin{array}{l}\text { Comprehensive analysis using a microarray } \\
\text { allowed selection of six miRNAs from } 1223 \\
\text { miRNAs }\end{array}$ \\
$\begin{array}{l}\text { Four species of miRNA were identified using } \\
\text { RT-qPCR in the sera of } 20 \text { subjects with } \\
\text { cervical cancer and } 10 \text { healthy subjects }\end{array}$ \\
$\begin{array}{l}\text { Verification was performed using multiple } \\
\text { specimens from the in the sera of } 45 \text { subjects } \\
\text { with cervical cancer, } 55 \text { subjects with CIN and } \\
31 \text { healthy subjects }\end{array}$ \\
\hline
\end{tabular}

Figure 1. The three-phase study design. miRNA, microRNA; RT-qPCR, reverse transcription-quantitative polymerase chain reaction; CIN, cervical intraepithelial neoplasia.

P-value of $<0.05$ was considered to indicate statistically significant differences.

\section{Results}

miRNA expression status in cervical cancer. The characteristics of the 131 subjects are summarized in Table I. The samples used in this study were obtained from subjects with CIN, subjects with cervical cancer undergoing surgery or biopsy and healthy volunteers, following informed consent. Table I shows the characteristics of the patients with cervical cancer. Total RNA was extracted from the serum. We first investigated the miRNA expression profiles using miRCURY LNA
microRNA array (Exiqon, Copenhagen, Denmark) in serum samples from 6 patients with cervical cancer and 6 healthy subjects to screen for candidate miRNAs associated with the development or progression of cervical cancer. The purpose of this analysis was to screen for a specific miRNA which may serve as a diagnostic or prognostic biomarker for cervical cancer patients. Initially, we compared miRNA expression in the serum of cervical cancer patients to that in the serum of healthy subjects. Of the 1,223 miRNAs compared in serum samples from cervical cancer patients and healthy controls using a miRCURY LNA microRNA array, 6 were found to exhibit a $>3.0$-fold change in their expression level $(\mathrm{P}<0.01)$.

To perform a technical selection of the array results, we analyzed 6 miRNAs in the serum of 20 patients with cervical cancer and 10 healthy subjects by RT-qPCR using TaqMan gene expression assays (Applied Biosystems). Among the 6 miRNAs examined, miR-485-5p, miR-1246, miR-1275 and particularly miR-1290, were found by RT-qPCR to be expressed at significantly higher levels in cervical cancer patients compared with healthy controls. As the difference in expression was particularly high for miR-1290, we focused on this miRNA in the next step.

Elevated miR-1290 in the serum of cervical cancer patients. We next determined whether miR-1290 could be detected in the serum and if it was more abundant in subjects with CIN and cervical cancer. We measured the miR-1290 concentration in 100 subjects with cervical neoplasia and 31 healthy controls. The expression level of miR-1290 was significantly elevated in the serum samples from subjects with cervical cancer, but not in those from subjects with CIN, compared with healthy controls $(\mathrm{P}<0.001$ and $\mathrm{P}>0.05$, respectively, t-test; 


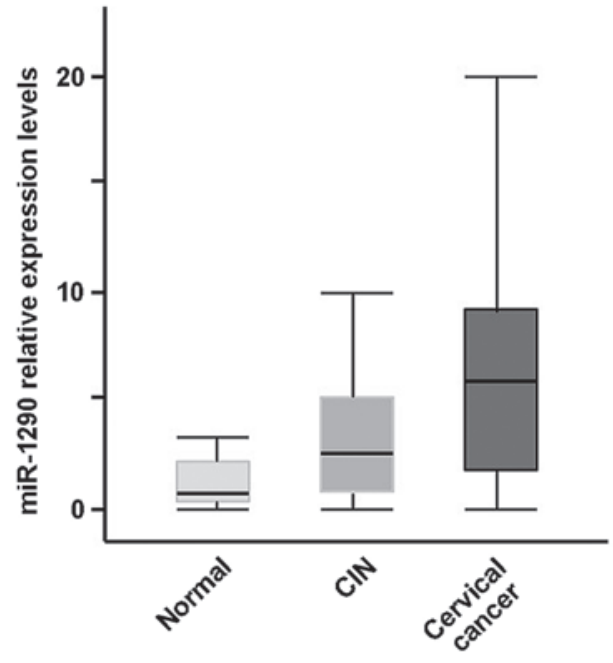

Figure 2. Circulating miR-1290 expression level in subjects with cervical cancer and cervical intraepithelial neoplasia (CIN) by quantitative polymerase chain reaction.

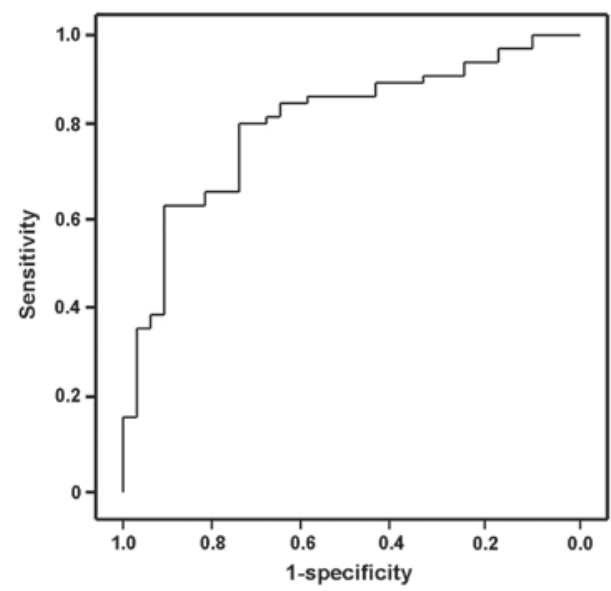

Figure 3. Area under the receiver operating characteristic curve for miR-1290 based on the quantitative polymerase chain reaction data. The cut-off level of plasma miR-1290 was 3.959; the sensitivity was $90.3 \%$ and the specificity was $62.2 \%$.

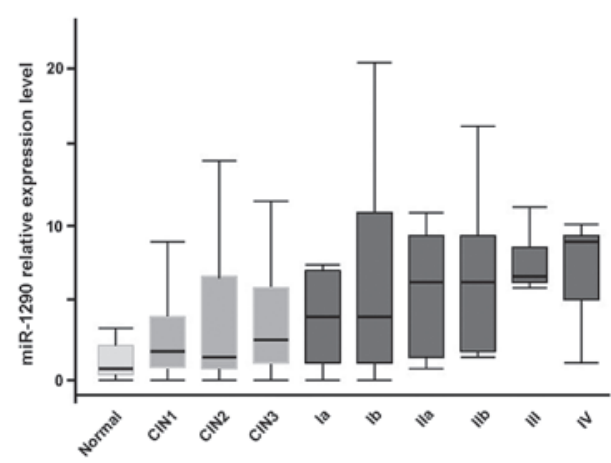

Figure 4. Circulating miR-1290 expression level in subjects with cervical cancer and cervical intraepithelial neoplasia (CIN) at various stages by quantitative polymerase chain reaction.

Fig. 2; miR-16 was used as a reference). The expression level of miR-1290 was significantly elevated in the serum of subjects with cervical cancer compared with that of CIN subjects

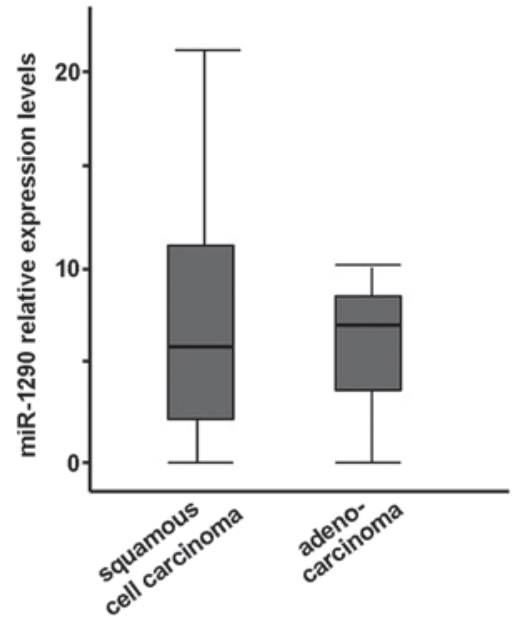

Figure 5. Circulating miR-1290 expression level in subjects with squamous cell carcinoma and adenocarcinoma of the cervix by quantitative polymerase chain reaction.

( $\mathrm{P}<0.01$, t-test; Fig. 2; miR-16 was used as a reference). The comprehensive analysis of the serum was performed using the multi-analyte serum miR-1290, which was significantly higher in subjects with cervical cancer compared with healthy subjects. The expression level of serum miR-1290 was the lowest in healthy subjects, higher in CIN subjects, and the highest in cervical cancer patients. The median serum miRNA-1290 expression level was 0.78 in healthy subjects, 2.46 in CIN subjects and 4.04 in cervical cancer patients $(\mathrm{P}<0.01$, Kruskal Wallis test).

The ROC curve indicated that the serum levels of miR-1290 may differentiate subjects with cervical cancer from healthy controls, with ROC curve areas of 0.7957 (95\% confidence interval: 0.6937-0.8977) (Fig. 3). With the cut-off at 3.959 (relative expression value), miR-1290 had a 90.3\% sensitivity for cervical cancer and a specificity of $62.2 \%$, compared with healthy controls.

Serum miR-1290 in subjects at each stage of cervical cancer. The median expression level of serum miR-1290 increased along with the stage of cervical cancer. The level in each group was as follows: Healthy group, 0.78; CIN1-2 group, 1.81; CIN3 group, 2.74; cervical cancer stage I group, 4.00; stage II group, 5.66; and stage III-IV group, $5.59(\mathrm{P}<0.01$, Kruskal-Wallis test, Fig. 4). There was no significant difference by histological type, i.e., SCC and adenocarcinoma (Welch's t-test, Fig. 5). In addition, there was no correlation between clinical data, such as smoking, SCC antigen level, carcinoembryonic antige level and lymph node metastasis, and miRNA expression (Mann-Whitney U test).

\section{Discussion}

As some biomarkers are able to detect cancer at early stages, they may improve patient survival; thus, they are key to the prognosis and diagnosis of cancer. SCC antigen is a tumor marker in cervical cancer, but the level of this marker is not increased during the early stages. Several clinicians have long stressed the need for novel biomarkers for early detection of cervical cancer. Abnormal expression of miRNAs 
has been reported in a number of cervical cancer tissues and cell lines (16). These miRNAs are able to promote cell proliferation and reduce apoptosis, affect cell invasion, and eventually contribute to the initiation and progression of cervical cancer (1). miRNAs are very stable and permit easy detection of specific types of cancer (17).

The identification of circulating miRNAs is one of the major scientific breakthroughs in recent years and it has revolutionized cell biology and medical science. Growth factors, such as cytokines, are the principal communication tools between cells, but communication by exosomes between the peripheral cells and cancer cells has also been noted to be significant (18). Circulating miRNAs function as 'extracellular communication RNAs' that play an important role in cell proliferation and differentiation (12). Blood-based miRNA profiling is not as reliable as tissue-based miRNA-profiling, but offers the potential for early, non-invasive, sensitive and specific cervical cancer detection and screening. Recently, several circulating miRNAs have been identified as potential serum biomarkers in different cancer types (19). These serum miRNAs may be effective as predictive biomarkers in cancer. Chen et al revealed that serum miRNA expression level is correlated with specific types of cancer, such as lung and colorectal cancer (17). Serum miRNAs are non-invasive biomarkers that may permit early detection of cancer.

We investigated the expression of serum miRNAs in healthy subjects, subjects with CIN and patients with cervical cancer using an miRNA microarray and RT-qPCR. The expression of serum miR-1290 was the lowest in healthy subjects, higher in CIN subjects, and the highest in cervical cancer patients. Moreover, the expression level of serum miR-1290 was higher in high-grade CIN subjects compared with that in subjects with lower-grade CIN. The expression level of miR-1290 tended to increase with advancing clinical stage of cervical cancer. High-risk HPV infection plays a central role in cervical carcinogenesis. These high-risk HPV serotypes are found in CIN, even in CIN1. HPV infection may increase miR-1290 expression directly or indirectly, since miR-1290 is already increased in CIN1. In order to normalize the miRNA expression level, miR-16 was used as an internal control, as previously reported $(13,14,20)$.

The miR-1290 gene is present in chromosome 1:19,223,565-19,223,642 (http://www.mirbase.org/). Some of the functions of miR-1290 have been previously reported. Wu et al suggested that miR-1290 is significantly upregulated in colon cancer tissues (21); moreover, they found that upregulation of miR-1290 impairs cytokinesis and leads to the formation of multinucleated cells in vitro and in vivo, while also resulting in Akt and nuclear factor- $\kappa \mathrm{B}$ activation, which maintains cell proliferation (21). Endo et al suggested that miR-1290 and its potential target genes, forkhead box protein A1 (FOXA1) and $\mathrm{N}$-acetyltransferase (NAT) 1, may be associated with the characteristics of estrogen receptor-positive breast cancer (22). FOXA1 is a forkhead family transcription factor, and arylamine NATs, known as drug- and carcinogen-metabolizing enzymes, transfer an acetyl group from acetyl coenzyme A to arylamines. They also suggested that B-cell lymphoma 2 (BCL2) and microtubuleassociated protein tau (MAPT) are potential targets of miR-1290 according to in silico analysis. BCL2 is an anti-apoptotic protein that exerts an antiproliferative effect through affecting cell cycle entry. MAPT binds to both the outer and the inner surfaces of microtubules, leading to tubulin assembly and microtubule stabilization (22). It has been reported that 6 miRNAs, including miR-1290, are upregulated in drug-sensitive cells following Y-Box protein 1 inhibition, but no differences in miRNA expression have been detected in multidrug-resistant gastric carcinoma cells (23). It has also been reported that 36 miRNAs, including miR-1290, circulate at higher levels in subjects with renal cell carcinoma compared with those in healthy controls (24).

We found that serum circulating miR-485-5p, miR-1246 and miR-1275, as well as miR-1290, were significantly higher in cervical cancer subjects compared with healthy controls. The expression of miR-485-5p and miR-1275 was significantly downregulated in hepatocelllular carcinoma (HCC) tissues $(25,26)$. In particular, miR-485-5p expression was inversely correlated with TNM stage and metastasis in HCC samples (25). Serum miR-1246 was significantly higher in primary colorectal and esophageal cancer patients compared with in healthy controls and significantly correlated with TNM stage $(27,28)$. Therefore, these three miRNAs (miR-485-5p, miR-1246 and miR-1275), may be biomarkers for cervical cancer with lower specificity.

In conclusion, serum miR-1290 appears to be a useful biomarker for the early detection of cervical cancer. It may reduce the need for invasive cervical biopsies and be useful in predicting the prognosis of cervical cancer. Larger studies are required to fully elucidate the role of miR-1290 in cervical cancer, and to determine whether serum miR-1290 may serve as a diagnostic marker or biomarker of treatment efficacy in this disease.

\section{Acknowledgements}

The authors would like to thank Ms. Chinatsu Yoneyama (Department of Obstetrics and Gynecology, Tokyo Medical University) and Dr Tomohiro Umezu (Department of Molecular Oncology, Institute of Medical Science, Tokyo Medical University) for providing invaluable experimental assistance. This study was supported by the Private University Strategic Research Based Support Project (grant no., S1311016) and Grants-in-Aids (grant no., 24592533) from the Ministry of Education, Culture, Sports, Science and Technology of Japan.

\section{References}

1. Li BH, Zhou JS, Ye F, Cheng XD, Zhou CY, Lu WG and Xie X: Reduced miR-100 expression in cervical cancer and precursors and its carcinogenic effect through targeting PLK1 protein. Eur J Cancer 47: 2166-2174, 2011

2. Yamamoto N, Kinoshita T, Nohata N, Itesako T, Yoshino $H$, Enokida H, Nakagawa M, Shozu M and Seki N: Tumor suppressive microRNA-218 inhibits cancer cell migration and invasion by targeting focal adhesion pathways in cervical squamous cell carcinoma. Int J Oncol 42: 1523-1532, 2013.

3. Bosch FX, Lorincz A, Muñoz N, Meijer CJ and Shah KV: The causal relation between human papillomavirus and cervical cancer. J Clin Pathol 55: 244-265, 2002.

4. Pisani P, Bray F and Parkin DM: Estimates of the world-wide prevalence of cancer for 25 sites in the adult population. Int $\mathrm{J}$ Cancer 97: 72-81, 2002.

5. McCredie MR, Sharples KJ, Paul C, Baranyai J, Medley G, Jones RW and Skegg DC: Natural history of cervical neoplasia and risk of invasive cancer in women with cervical intraepithelial neoplasia 3: A retrospective cohort study. Lancet Oncol 9: 425-434, 2008 
6. Kato H Morioka H, Aramaki S and Torigoe T: Radioimmunoassay for tumor-antigen of human cervical squamous cell carcinoma Cell Mol Biol Incl Cyto Enzymol 25: 51-56, 1979.

7. Kato H: Squamous cell carcinoma antigen. In: Serological cancer Markers. Sell S (ed). Humana Press, Totowa, pp437-pp451, 1992

8. Lee RC, Feinbaum RL and Ambros V: The C. elegans heterochronic gene lin-4 encodes small RNAs with antisense complementarity to lin-14. Cell 75: 843-854, 1993.

9. Lytle JR, Yario TA and Steitz JA: Target mRNAs are repressed as efficiently by microRNA-binding sites in the 5'UTR as in the 3'UTR. Proc Natl Acad Sci USA 104: 9667-9672, 2007.

10. Esquela-Kerscher A and Slack FJ: Oncomirs-microRNAs with a role in cancer. Nat Rev Cancer 6: 259-269, 2006

11. Lewis BP, Burge CB and Bartel DP: Conserved seed pairing, often flanked by adenosines, indicates that thousands of human genes are microRNA targets. Cell 120: 15-20, 2005.

12. Valadi H, Ekström K, Bossios A, Sjöstrand M, Lee JJ and Lötvall JO: Exosome-mediated transfer of mRNAs and microRNAs is a novel mechanism of genetic exchange between cells. Nat Cell Biol 9: 654-659, 2007.

13. Ohyashiki K, Umezu T, Yoshizawa S, Ito Y, Ohyashiki M, Kawashima H, Tanaka M, Kuroda M and Ohyashiki JH Clinical impact of down-regulated plasma miR-92a levels in non-Hodgkin's lymphoma. PLoS One 6: e16408, 2011.

14. Schrauder MG, Strick R, Schulz-Wendtland R, Strissel PL, Kahmann L, Loehberg CR, Lux MP, Jud SM, Hartmann A, Hein A, et al: Circulating micro-RNAs as potential blood-based markers for early stage breast cancer detection. PLoS One 7: e29770, 2012.

15. Kanda Y: Investigation of the freely available easy-to-use software 'EZR' for medical statistics. Bone Marrow Transplant 48: 452-458, 2013.

16. Martinez I, Gardiner AS, Board KF, Monzon FA, Edwards RP and Khan SA: Human papillomavirus type 16 reduces the expression of microRNA-218 in cervical carcinoma cells Oncogene 27: 2575-2582, 2008.

17. Chen X, Ba Y, Ma L, Cai X, Yin Y, Wang K, Guo J, Zhang Y, Chen J, Guo X, et al: Characterization of microRNAs in serum: A novel class of biomarkers for diagnosis of cancer and other diseases. Cell Res 18: 997-1006, 2008.

18. Al-Nedawi K, Meehan B, Micallef J, Lhotak V, May L, Guha A and Rak J: Intercellular transfer of the oncogenic receptor EGFRvIII by microvesicles derived from tumour cells. Nat Cell Biol 10: 619-624, 2008.
19. Allegra A, Alonci A, Campo S, Penna G, Petrungaro A, Gerace D and Musolino C: Circulating microRNAs: New biomarkers in diagnosis, prognosis and treatment of cancer (review). Int J Oncol 41: 1897-1912, 2012.

20. Xu J, Cao Z, Liu W, You L, Zhou L, Wang C, Lou W, Sun B, Miao Y, Liu X, et al: Plasma miRNAs effectively distinguish patients with pancreatic cancer from controls: A multicenter study. Ann Surg Jun 25: (Epub ahead of print), 2015.

21. Wu J, Ji X, Zhu L, Jiang Q, Wen Z, Xu S, Shao W, Cai J, Du Q, Zhu Y and Mao J: Up-regulation of microRNA-1290 impairs cytokinesis and affects the reprogramming of colon cancer cells. Cancer Lett 329: 155-163, 2013.

22. Endo Y, Toyama T, Takahashi S, Yoshimoto N, Iwasa M, Asano T, Fujii Y and Yamashita H: miR-1290 and its potential targets are associated with characteristics of estrogen receptor $\alpha$-positive breast cancer. Endocr Relat Cancer 20: 91-102, 2013.

23. Belian E, Kurucz R, Treue D and Lage H: Effect of YB-1 on the regulation of microRNA expression in drug-sensitive and drug-resistant gastric carcinoma cells. Anticancer Res 30: 629-633, 2010

24. Wulfken LM, Moritz R, Ohlmann C, Holdenrieder S, Jung V, Becker F, Herrmann E, Walgenbach-Brünagel G, von Ruecker A, Müller SC and Ellinger J: MicroRNAs in renal cell carcinoma: Diagnostic implications of serum miR-1233 levels. PLoS One 6: e25787, 2011.

25. Sun X, Liu Y, Li M, Wang M and Wang Y: Involvement of miR-485-5p in hepatocellular carcinoma progression targeting EMMPRIN. Biomed Pharmacother 72: 58-65, 2015.

26. Fawzy IO, Hamza MT, Hosny KA, Esmat G, El Tayebi HM and Abdelaziz AI: miR-1275: A single microRNA that targets the three IGF2-mRNA-binding proteins hindering tumor growth in hepatocellular carcinoma. FEBS Lett 589: 2257-2265, 2015.

27. Ogata-Kawata H, Izumiya M, Kurioka D, Honma Y, Yamada Y, Furuta K, Gunji T, Ohta H, Okamoto H, Sonoda H, et al: Circulating exosomal microRNAs as biomarkers of colon cancer. PLoS One 9: e92921, 2014.

28. Takeshita N, Hoshino I, Mori M, Akutsu Y, Hanari N, Yoneyama Y, Ikeda N, Isozaki Y, Maruyama T, Akanuma N, et al: Serum microRNA expression profile: miR-1246 as a novel diagnostic and prognostic biomarker for oesophageal squamous cell carcinoma. Br J Cancer 108: 644-652, 2013. 